\title{
Advanced Midzonal Iris Pigment Epithelial Cyst Managed by Aspiration Deflation
}

\author{
Jerry A. Shields Samuel J. Fallon Charlotte Nelle Shields Sara E. Lally \\ Carol L. Shields \\ Ocular Oncology Service, Wills Eye Hospital, Thomas Jefferson University, Philadelphia, PA, USA
}

\section{Keywords}

Eye · Iris · Cyst · Midzonal cyst · Iris pigment epithelium .

Optical coherence tomography

\begin{abstract}
Background: There are several cysts that can occur in the iris. The midzonal (retroiridic) iris pigment epithelial (IPE) cyst is important because it can simulate melanoma of the ciliary body. However, it is frequently asymptomatic and requires no treatment. Methods: A 37-year-old asymptomatic woman with visual acuity of 20/20 in each eye was found to have an elevated mass behind the left iris, presumed to be melanoma, and was referred for management. On our examination, there was a typical midzonal IPE cyst in the left eye (OS), requiring observation without intervention. After 4 years, she developed progressive visual loss to 20/40 OS and cyst enlargement was noted, obstructing most of the visual axis and causing partial lens subluxation. Translimbal fine needle aspiration deflation with a 30-G needle was performed. $\boldsymbol{R} \boldsymbol{e}$ sults: Immediately at aspiration, the cyst demonstrated collapse, disappearing behind the iris stroma, and the lens subluxation resolved. Visual acuity returned to 20/20 OS and has remained stable without recurrence for 3 years. Conclusion: Although most midzonal IPE cysts are asymptomatic, this
\end{abstract}

\section{KARGER}

(c) 2017 S. Karger AG, Basel

E-Mail karger@karger.com

www.karger.com/oop case showed progressive enlargement with visual loss and was managed with translimbal aspiration. Symptomatic midzonal IPE cysts can be deflated with translimbal aspiration rather than more aggressive surgical excision.

(c) 2017 S. Karger AG, Basel

\section{Introduction}

Iris cysts are classified into primary and secondary types with several subtypes of each [1-9]. Primary iris cysts are further subclassified into cysts of the iris stroma and cysts of the iris pigment epithelium. Iris stromal cysts demonstrate more aggressive features, particularly in young infants, with large sizes, endothelial adhesion, angle closure, glaucoma, and severe photophobia due to microruptures, often requiring interventional therapy [8]. By contrast, iris pigment epithelial (IPE) cysts are classified into central, midzonal, peripheral, free-floating, and dislodged, with a more quiescent stationary course, and they rarely require intervention $[5,7]$.

The midzonal IPE cyst can be clinically challenging to diagnose, as it is often hidden behind the iris as a round brown mass, simulating ciliary body melanoma. A midzonal IPE cyst either cannot be seen or may be round in 

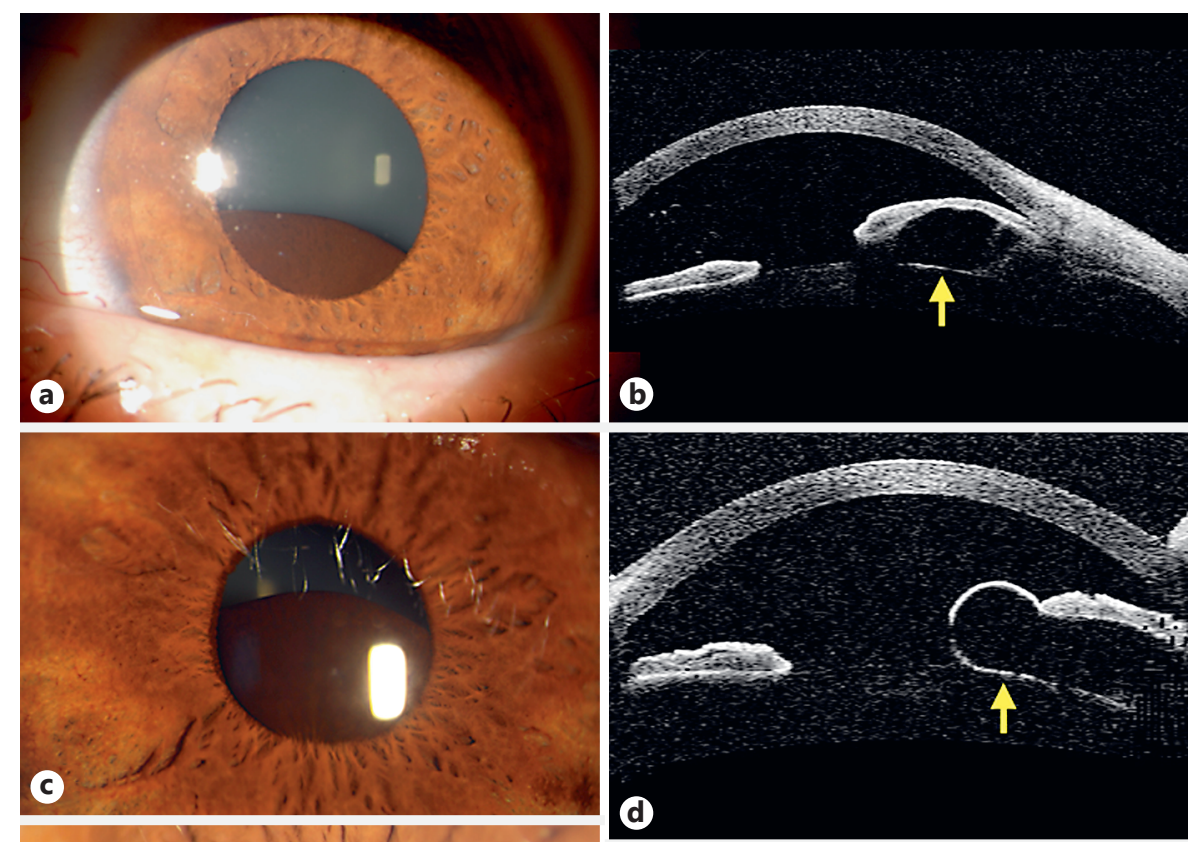

Fig. 1. Documented enlargement of a midzonal iris pigment epithelial (IPE) cyst, managed with aspiration deflation. $\mathbf{a}, \mathbf{b}$ September 2009. Smooth, brown IPE cyst visualized on anterior segment optical coherence tomography (AS-OCT; b) demonstrating anterior displacement of the iris stroma from the posterior, thin-walled cyst (arrow). c, d September 2013. The IPE cyst had demonstrated enlargement clinically and covered two-thirds of the pupil with enlargement (d, arrow) on AS-OCT. e, f March 2014. The IPE cyst now occupied approximately $70 \%$ of the pupil. On ASOCT (f), the bulbous cyst (arrow) protruded into the visual axis. g, h April 2014. Following aspiration deflation, the IPE cyst was no longer clinically visible and the pupil was clear. AS-OCT $(\mathbf{h})$ revealed only a small cyst remnant (arrow) with a flat iris.
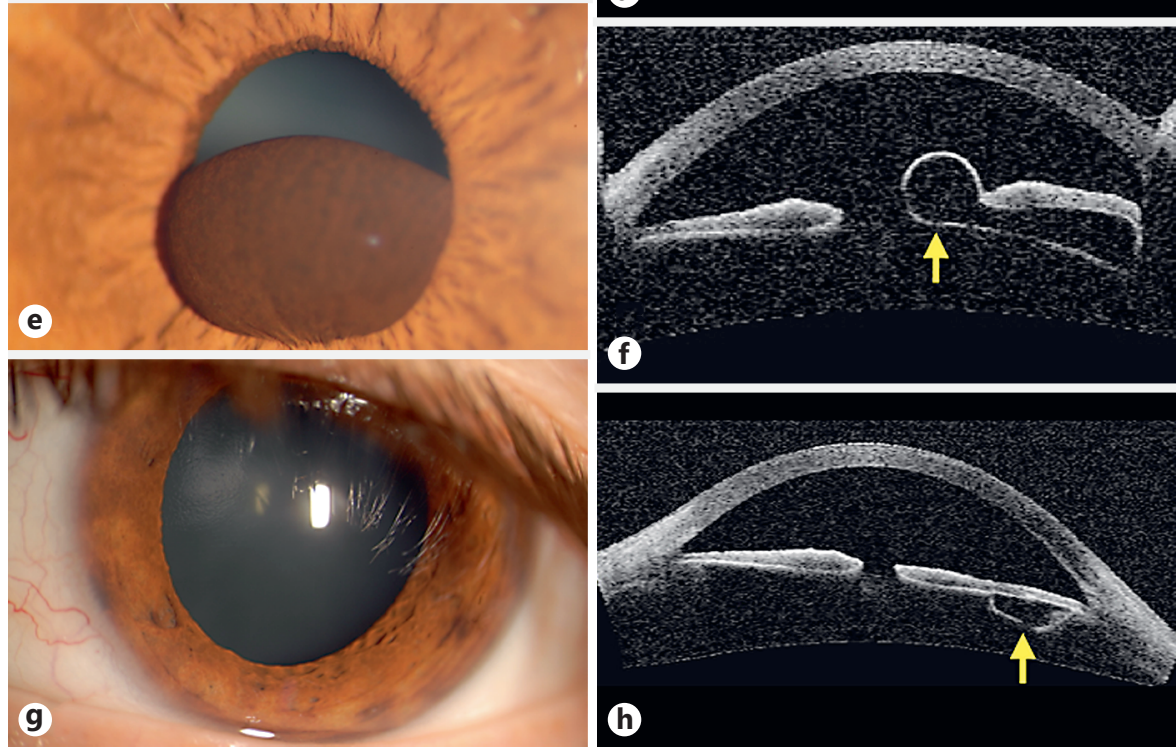

the nondilated state and more elongated or fusiform following pupillary dilation. This brown-colored lesion demonstrates further elongation with further dilation, an evolving picture not seen with melanoma. These clinical features, in combination with anterior segment imaging on ultrasound biomicroscopy (UBM) and anterior segment optical coherence tomography (AS-OCT), help to differentiate midzonal IPE cyst from ciliary body melanoma. Herein, we describe a midzonal IPE cyst that enlarged over 4 years and caused loss of vision, but responded to needle aspiration deflation.

Advanced Midzonal IPE Cyst Managed by Aspiration Deflation

\section{Case Report}

In September 2009, a 37-year-old asymptomatic woman was referred for evaluation and management of a possible ciliary body melanoma in the left eye (OS). On our examination, visual acuity was 20/20 in each eye (OU) and intraocular pressures were normal OU. The right eye was unremarkable. The left eye demonstrated an asymptomatic, smooth, uniformly brown, rounded mass in the inferior retroiridic location simulating melanoma; however, following pupillary dilation, the mass became more fusiform and occluded the inferior one-third of the pupil (Fig. 1a). There were no sentinel blood vessels. Imaging with UBM and AS-OCT demonstrated anterior displacement of the iris stroma by a smooth, thinwalled cyst, with no evidence of a solid mass (Fig. 1b). A diagnosis 
of midzonal IPE cyst without a solid component was made and observation was recommended.

The patient returned in September 2013 with a complaint of blurred vision and ocular discomfort OS. Visual acuity was 20/20 in the right eye (OD) and 20/40 OS, with normal intraocular pressure OU. Examination revealed progressive enlargement of the IPE cyst OS, occluding approximately two-thirds of the pupil (Fig. 1c). Imaging with UBM and AS-OCT again confirmed a cystic structure that overhung the pupil (Fig. 1d).

After extensive counseling, she declined surgical intervention, despite vision loss. She was prescribed phenylephrine $2.5 \%$ eye drops twice daily for 3 weeks by her local physician, with no relief. In March 2014, she complained of slight visual impairment OS, with visual acuity of 20/40 probably because of cyst encroachment into the visual axis. The intraocular pressure was normal. Examination revealed the IPE cyst now occluded about $70 \%$ of the pupillary aperture, with new plump overhang into the anterior chamber (Fig. 1e). AS-OCT showed further extension of the cyst across the pupil (Fig. 1f). The patient agreed to surgical intervention.

Using local retrobulbar anesthesia and the operating microscope, we passed a $30-\mathrm{G}$ needle through the corneoscleral limbus inferotemporally and directed it through the cyst wall inferiorly at the pupillary margin inferiorly, avoiding the visual axis. Slow aspiration into a $10-\mathrm{mL}$ syringe was performed, and the cyst promptly collapsed and disappeared behind the iris stroma.

On examination 2 months following the aspiration deflation, visual acuity was 20/20 OU, the ocular discomfort had resolved, and the cyst was not visible, even following wide dilation of the pupil (Fig. 1g). On AS-OCT, a small remnant behind the iris stroma was observed (Fig. 1h). The patient has remained comfortable with stable visual acuity and without cyst recurrence for 3 years following aspiration.

\section{Discussion}

In a comprehensive analysis of 3,680 iris lesions, solid tumors were found in 2,912 (79\%) and cystic lesions in 768 (21\%) [9]. Analysis of the various IPE cysts revealed central (7\%), midzonal (28\%), peripheral (63\%), and freefloating/dislodged $(2 \%)$ types $[7,9]$. In that cohort, there were 188 eyes with stromal IPE cysts, of which 29 (15\%) were those of children ( $0-20$ years), 30 (16\%) were those of young adults (21-40 years), 50 (27\%) were those of mid-adults (41-60 years), and 79 (42\%) were those of older adults ( $>61$ years) [7]. Our patient was 37 years old at presentation, an age more commonly associated with peripheral (75\%) rather than midzonal (12\%) IPE cysts [7].

Most midzonal IPE cysts are located posterior to the iris stroma and often not clinically visible. By meticulous slit lamp biomicroscopy, an IPE cyst is suspected if there is an anterior bulge of the otherwise normal overlying iris stroma. Following pupillary dilation, some IPE cysts are visible, including the central and midzonal types. Often, the peripheral type or dislodged lesions require further evaluation with gonioscopy to reach behind the iris stroma or into the anterior chamber angle for visualization of the cyst.

The midzonal IPE cyst is a particularly interesting lesion, since it closely simulates ciliary body melanoma, as in this case, prompting referral. A midzonal cyst can be partly visualized with a bulbous, smooth, uniform, brown surface behind the iris, quite similar to melanoma. However, midzonal IPE cysts become stretched from round to elongated following pupillary dilation. This feature, along with the lack of sentinel vessels, lack of a transillumination shadow, and imaging features of a cystic mass, serves to confirm the diagnosis. Most midzonal IPE cysts are asymptomatic and require no treatment.

This case was unusual, since most IPE cysts remain stable. Midzonal cysts classically appear unilobular or multilobular, rarely covering the visual axis or affecting visual acuity. In our case, the midzonal IPE cyst extended approximately one-third over the pupillary aperture and later enlarged to more than two-thirds the pupillary diameter, causing low-grade ocular discomfort and vision loss.

Most IPE cysts are asymptomatic and require no treatment. Occasionally, central cysts may require deflation, but this is rarely necessary for other types of cyst. In this case, needle aspiration deflation allowed for relocation of the partially subluxated lens and retention of visual acuity. This approach for a symptomatic midzonal IPE cyst can achieve rapid resolution of symptoms when compared with surgical removal, laser photocoagulation, or other methods.

In summary, we described a rare midzonal IPE cyst that demonstrated enlargement and caused ocular discomfort and visual loss. It was successfully managed with aspiration deflation.

\section{Statement of Ethics}

Our institutional review board does not require approval for data from a single patient.

\section{Disclosure Statement}

Support was provided by the Eye Tumor Research Foundation, Philadelphia, PA, USA (J.A.S. and C.L.S.). The funders had no role in the design and conduct of the study; in the collection, analysis, and interpretation of the data; and in the preparation, review, or approval of the manuscript. J.A.S. has had full access to all the data in the study and takes responsibility for the integrity of the data and the accuracy of the data analysis. No conflicting relationship exists for any author. 


\section{References}

1 Shields JA: Primary cysts of the iris. Trans Am Ophthalmol Soc 1981;79:771-809.

2 Lois N, Shields CL, Shields JA, et al: Primary cysts of the iris pigment epithelium: clinical features and natural course in 234 patients. Ophthalmology 1998;105:1879-1885.

3 Shields JA, Shields CL, Lois N, et al: Iris cysts in children: classification, incidence, and management. The 1998 Torrence A Makley Jr Lecture. Br J Ophthalmol 1999;83:334-338.

4 Shields CL, Shields PW, Manalac J, et al: Review of cystic and solid tumors of the iris. Oman J Ophthalmol 2013;6:159-164.
5 Lois N, Shields CL, Shields JA, et al: Primary cysts of the iris pigment epithelium: clinical features and natural course in 234 patients. Ophthalmology 1998;105:1879-1885.

6 Bianciotto C, Shields CL, Guzman JM, et al: Assessment of anterior segment tumors with ultrasound biomicroscopy versus anterior segment optical coherence tomography. Ophthalmology 2011;118:1297-1302.
7 Shields JA, Shields CL: Cysts of the iris pigment epithelium. What is new and interesting? The 2016 Jose Rizal International Medal Lecture. Asia Pac J Ophthalmol (Phila) 2017; 6:64-69.

8 Shields CL, Arepalli S, Lally EB, et al: Iris stromal cyst management with absolute alcoholinduced sclerosis in 16 patients. JAMA Ophthalmol 2014;132:703-708.

9 Shields CL, Kancherla S, Patel J, et al: Clinical survey of 3,680 iris tumors based on patient age at presentation. Ophthalmology 2012; 119:407-414 